\title{
CHILD Syndrome
}

\author{
Sejal Neel*, Neel Kanth, Raveet Kumar \\ Department of Pediatrics, Sindh Government Children Hospital, Karachi, Pakistan.
}

\begin{abstract}
CHILD syndrome is a rare X-linked genetic disorder caused by mutation of NSDHL (NADPH steroid dehydrogenase-like protein) at Xq28 gene. The mutation causes the defective synthesis of cholesterol, which is an important constituent of viscera, hormones etc. The predominantly it affects the musculoskeletal with a tendency to involve the heart, brain etc. Strikingly the signs and symptoms of this syndrome involve only one half of the body. CHILD syndrome is commonly identified in the females. We report a case of 5-month-old female who presented to us at Pediatric outpatient department. To our knowledge this is the first reported case of CHILD syndrome in Pakistan. The exceptionally rare presentation prompted us to report this case.
\end{abstract}

Keywords: CHILD syndrome, NSDHL mutation, Ichthyosis, Genetic disorder, Alopecia, Abortion, Sporadic.

\section{INTRODUCTION}

CHILD syndrome, an acronym for Congenital Hemidysplasia with Icthyosiform Erythroderma and Limb Defect, is a rare $\mathrm{X}$-linked dominant genetic disorder. Since its first identification in 1903, to the date approximately 60 cases are reported in the literature. Due to mode of inheritance, it has predilection towards female heterozygous and proven nearly lethal in male hemizygous patients [1]. Female to male ratio is 19:1 [2].

Clinical features result from mutation of NSDHL gene, which is involved, in cholesterol synthesis. CHILD syndrome is multisystem disorder, which primarily tends to have skin and skeletal deformities. Vulva, axillae and gluteal folds are the most commonly affected sites [3]. The lateralization of epidermal nevus to one side of body, usually right side, has well appreciable mid-line skin demarcation [3]. CHILD syndrome commonly accompanies ipsilateral limb and occasionally visceral malformations $[1,3]$.

To the best of our knowledge, being the only case of CHILD syndrome in Pakistan, additional findings of hydronephrosis and normochromic-normocytic anemia have not been documented in the literature.

\section{CASE PRESENTATION}

A five months old female, 7th born child of the family, came to the Pediatric outpatient department with red colored scaly rash on left side of the body, strict midline demarcation, and swelling of left leg and foot. She was born by spontaneous vaginal delivery without any adverse birth events. Antenatal history was unremarkable. Mother denied any history of miscarriages or abortion. There was no additional significant finding on the systemic examination. Family history was non-contributory.

\footnotetext{
*Address correspondence to this author at the Department of Pediatrics, Sindh Government Children Hospital, Karachi, Pakistan.

E-mail: sejal.neel@hotmail.com
}

On Examination, left side of the body revealed a thickened red rash with brown scaly lesions involving left arm and leg in addition to the half of trunk and perineum. Left labia majora was hypertrophied with inguinal folds showing infected skin lesions. The child's left hand was swollen. Left foot showed ectrodactyly (Fig.1) and involvement of complete half of the body (Fig. 1 and 2). Face and neck were spared bilaterally (Fig. 1 and 3). No associated alopecia. No nail involvement was seen. Examination of cardiovascular system, lungs, brain and other viscera were normal.

Laboratory investigations revealed normochromic normocytic anemia. Renal ultrasound showed mild left sided hydronephrosis. Skeletal survey concluded normal bone density with reduced length of long bones of left lower limb. Liver and kidney function tests, and other blood biochemistry were within normal limits. On basis of a well evident clinical picture this patient was diagnosed as CHILD syndrome. Due to limitations genetic studies were not done. She was prescribed skin emollients and topical antibiotics for macerated area over the inguinal folds. Regular follow ups were advised.

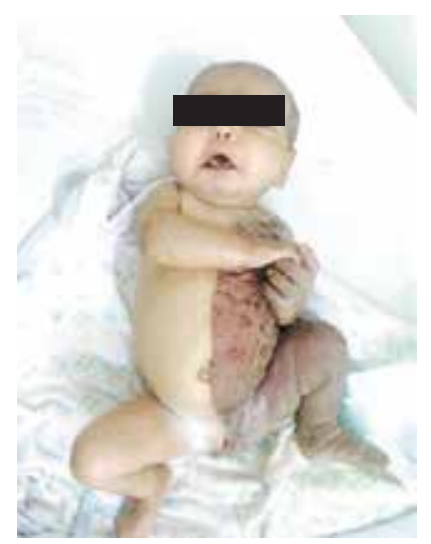

Fig. (1). Showing Left Foot Ectrodactyly, Complete Half of Body is involved with Sharp Demarcation and Face is Spared. 


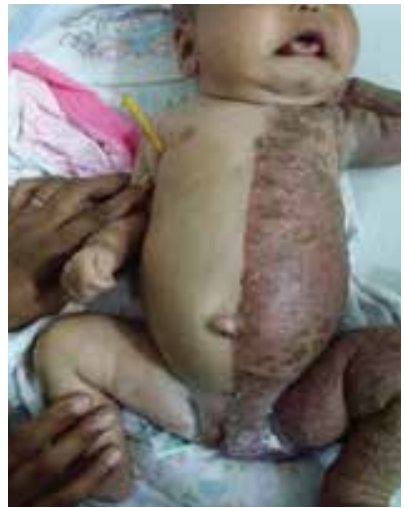

Fig. (2). Sharp Demarcation of in the Mid Line with Only Half of Body Being involved.

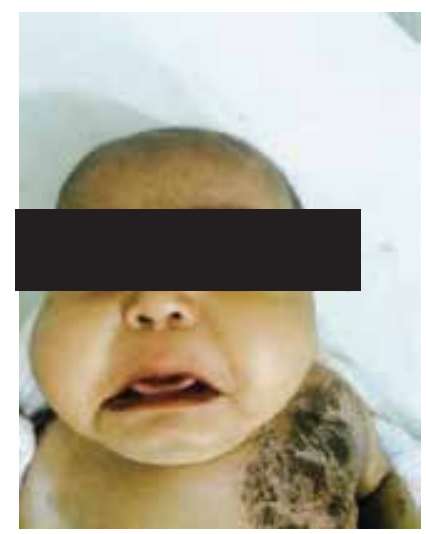

Fig. (3). Face is Bilaterally Spared and Does not have Ichthyosiform Lesion.

\section{DISCUSSION}

Cholesterol is an important component of cell membranes and organelles inside the cells, and hedge-hog signaling [4, 5]. There are several enzymes which play role in the biosynthesis of cholesterol, and mutation of both the pre- sequel and post-sequel has been reported. There are of 7 seven post-sequel mutations are so far identified and one of such mutation includes NSDHL $(\mathrm{NAD}(\mathrm{P}) \mathrm{H}$ steroid dehydrogenase-like protein) gene, which located at Xq2 [1, 6, 7]. There are 20 different types of mutations identified so far in different cases, including point mutation, missense, non-sense, stop mutations, etc. [8-10]. Loss of function of NSDHL gene leads to the reduced functions of the 3-betahydroxysterol dehydrogenase enzyme [1]. This enzyme complex catalyzes the removal of c-4 methyl group from lansoprazole in cholesterol biosynthesis pathway [11]. Besides, low cholesterol in body, it also leads to accumulation of toxic cholesterol metabolites. Impaired cholesterol processing also causes abnormal sonic hedge-hog signaling, which plays special role in the patterning of embryos [1].
Together, all this impaired functioning leads to the CHILD syndrome.

Like our patient majority of cases clinically present at birth or within few initial weeks of their life $[3,12]$. There are few cases reported with delayed presentations, like at the age of 9 years. Although erythematous hyperkeratotic verrucous scaly plaques of CHILD syndrome tend to be ipsilateral. But, there have been cases nearly symmetric erythematous plaques [1]. Most cases account for right-sided presentations. However, exception to this has been reported in nearly 20 cases. Left-sided patients have severe visceral presentation and therefore has higher probabilities of early death [12]. With severe visceral involvement there are reported cases with ipsilateral hypoplasia of spinal cord, cranial nerves, brain, adrenal gland, lung, ovary, fallopian tubes, etc. [1]. Unlike cases in literature with lymphocytosis or absent kidney with contralateral renal hypertrophy, our patient had normochromic-normocytic anemia and left sided-hydronephrosis $[2,8]$.

Skin nevus of CHILD syndrome tends not to exacerbate and complete remission has been found with the regular use pathogenesis-based therapies [13]. Topical applications like lotions or ointment of lovastatin or simvastatin as monotherapy or along with cholesterol has shown reduction in inflammation, skin thickening, scaling and lead to complete reversion of CHILD nevus $[4,11,14]$. Antibiotics play important role during superimposed infections. Treatment with emollients (other then statins) and topical retinoids are of limited benefit. Apart from medications, dermabrasion and skin grafting have also been tried with positive outcomes [11]. With respected to the complications, cardiovascular outcomes are leading cause of mortality in this condition. There is also a case squamous cell carcinoma, reported as a consequence of CHILD syndrome in 33-year-old female [15].

Due to limitation, genetic and molecular diagnosis could not be obtained. Germinal mosaicism is difficult to exclude [6]. Even the lethal outcomes in the male according to the literature are due to mosaicism in NSDHL gene mutation [1]. In the CHILD syndrome the diversity of symptoms and its severity is assumed due to skewed post-zygotic $\mathrm{X}$-inactivation [1]. Literature also suggests that similar mutations can cause either of the left or right sided illness. And it may be the diversity in the patterns of X chromosome inactivation which leads to the divers clinical presentations and severity of the syndrome $[11,16]$. Beyond literature review which is mainly based on observations, the exact understanding regarding fatality of syndrome could not be identified [12]. Majority of the researchers suggest that risk of CHILD syndrome in subsequent pregnancies is near to general population at risk and parents are the proband for this genetic condition $[6,12]$. Based on one exception where 3 generations of single family had had diagnosis of CHILD 
syndrome, there is an assumption that several cases who are currently identified sporadic, may indeed have familial background [17]. Another rare syndrome which is caused by mutations in the similar NSDHL gene, called as CK syndrome, is known to have familial background due to its presence in the 5 generations of family [18].

\section{CONCLUSION}

Although, CHILD syndrome is rarely identified condition. But delay in the diagnosis could be minimized by a thorough understanding of its classic clinical presentation. A timely initiation of treatment with appropriate hygienic conditions for deformed skin to reduce superimposed infections can reduce long term complications and improve outcomes. It is more important in case of left sided presentation as patients tend to have severe visceral involvement and early death, like in our patient. Although exact cause of death is not known as clinically it did not have any visceral complications at presentation. In addition to that, irrespective of sporadic occurrence CHILD syndrome, the genetic counselling should always be a part of conversation with the families $[5,11]$.

\section{CONFLICT OF INTEREST}

Declared none.

\section{ACKNOWLEDGEMENTS}

Declared none.

\section{REFERENCES}

[1] Shawky RM, Elsayed SM, Amgad H. Autosomal recessive ichthyosis with limb reduction defect: A simple association and not CHILD syndrome. Egypt J Med Hum Genetics 2016; 17(3): 255-8. DOI: 10.1016/j.ejmhg.2015.08.009

[2] Happle R, Koch H, Lenz W. The CHILD syndrome. Eur J Pediatr 1980; 134(1): 27-33. DOI: 10.1007/BF00442399

[3] Christiansen AG, Koppelhus U, Sommerlund M. Skin abnormalities in CHILD syndrome successfully treated with pathogenesis-based therapy. Acta Derm Venereol 2015; 95(6): 752-3. DOI: $10.2340 / 00015555-2044$

[4] Asch S, Sugarman JL. Epidermal nevus syndromes: New insights into whorls and swirls. Pediatr Dermatol 2018; 35(1): 21-9. DOI: $10.1111 /$ pde. 13273

[5] Stottmann RW, Turbe-Doan A, Tran P, et al. Cholesterol metabolism is required for intracellular hedgehog signal transduction in vivo. PLoS Genet 2011; 7(9): e1002224.

DOI: 10.1371/journal.pgen.1002224

[6] Schmidt-Sidor B, Obersztyn E, Szymańska K, et al. Brain and cerebellar hemidysplasia in a case with ipsilateral body dysplasia and suspicion of CHILD syndrome. Folia Neuropathol 2008; 46(3): 232-7.
[7] Cunningham D, Swartzlander D, Liyanarachchi S, et al. Changes in gene expression associated with loss of function of the NSDHL sterol dehydrogenase in mouse embryonic fibroblasts. J Lipid Res 2005; 46(6): 1150-62.

DOI: 10.1194/jlr.M400462-JLR200

[8] Chander R, Varghese B, Jabeen M, et al. CHILD syndrome with thrombocytosis and congenital dislocation of hip: A case report from India. Dermatol Online J 2010; 16(8): 6.

[9] Mi XB, Luo MX, Guo LL, et al. CHILD syndrome: Case report of a chinese patient and literature review of the NAD [P] $\mathrm{H}$ steroid dehydrogenase-like protein gene mutation. Pediatr Dermatol 2015; 32(6): e277-82. DOI: 10.1111/pde.12701

[10] Bornholdt D, König A, Happle R, et al. Mutational spectrum of NSDHL in CHILD syndrome. J Med Genet 2005; 42(2): e17. DOI: $10.1136 / j m g .2004 .024448$

[11] Yang Z, Hartmann B, Xu Z, et al. Large Deletions in the NSDHL gene in two patients with CHILD syndrome. Acta Derm Venereol 2015; 95(8): 1007-8.

DOI: $10.2340 / 00015555-2143$

[12] Dara S. CHILD syndrome: A rare genetic disorder. Am J Adv Nurs Res 2017; 4(2): 80-3.

[13] Seeger MA, Paller AS. The role of abnormalities in the distal pathway of cholesterol synthesis in the Congenital Hemidysplasia with Ichthyosiform erythroderma and Limb Defects (CHILD) syndrome. Biochim Biophys Acta (BBA)-Mol Cell Biol Lipids 2014; 1841(3): 345-52.

DOI: 10.1016/j.bbalip.2013.09.006

[14] Bajawi SM, Jafarri SA, Buraik MA, et al. Pathogenesis-based therapy: Cutaneous abnormalities of CHILD syndrome successfully treated with topical simvastatin monotherapy. JAAD Case Rep 2018;4(3): 232-4.

DOI: $10.1016 /$ j.jdcr.2017.11.019

[15] Jacyk WK, La Cock A. Squamous cell carcinoma arising in CHILD syndrome. J Eur Acad Dermatol Venereol 2006; 20(3): 311-3. DOI: 10.1111/j.1468-3083.2006.01411.x

[16] Hummel M, Cunningham D, Mullett CJ, et al. Left-sided CHILD syndrome caused by a nonsense mutation in the NSDHL gene. Am J Med Genet Part A 2003; 122(3): 246-51. DOI: 10.1002/ajmg.a.20248

[17] Bittar M, Happle R, Grzeschik KH, et al. CHILD syndrome in 3 generations: The importance of mild or minimal skin lesions. Arch Dermatol 2006;142(3): 348-51.

DOI: $10.1001 /$ archderm.142.3.348

[18] Preiksaitiene E, Caro A, Benušienė E, et al. A novel missense mutation in the NSDHL gene identified in a Lithuanian family by targeted next-generation sequencing causes CK syndrome. Am J Med Genet Part A 2015; 167(6): 1342-8.

DOI: 10.1002/ajmg.a.36999 